\title{
INVESTIGATION OF LEADED PETROL AND DIESEL ADULTERATION IN SRI LANKA
}

\author{
DR Kulatunga and KRR Mahanama \\ Department of Chemistry, Univers ty of Colombo, Colombo 03
}

Addition of adulterants to expensive consumer procucts is a common problem. Similarity of the physical and /or chemical properties of the consumer product to the adulterant is the biggest challenge in the identification of the adulterant. However, the selection of a noticeable difference of the adulterant to the consumer product can be utilized for the routine analysis of such an adulterant. The success of a routine analysis depends on several factors, which included simplicity, cost and the time for analysis.

In Sri Lanka, both petrol and diesel are believed to be added with low cost petroleum-based chemicals. The inclusion of cheaper adulterants increases with the ever-rising demand and cost of the fuels. This research project was initiated to inv estigate a suitable procedure in identifying the petroleum-based adulterants in commercially available petrol and diesel. Both these fuels are not pure compounds and they contain different distillation fractions of crude oil. Hence, the chemical constituents in these two fuels are diflerent but they have the similar chemical characteristics. Ploycyclic aromatic hydrocarbons (PAH), a group of fluorescence active compounds, are present in the petroleum base fuels. But, the PAH content in each of the fuel type varies with the boiling range of the fuel.

Synchronous fluorescence scan (SFS) seems to be a promising technique in identifying fluorescence active compounds in such samples. Unlike in conventional fluorescence spectroscopy, in SFS both the excitation and emission wavelengths are scanned keeping the difference of the wavelengths to a constant value We have investigated the optimal SFS conditions for unadulterated kerosene, diesel and leaded petrol. Then the analysis were carried out for the mixtures of leaded petrol + kerosene and diesel + kerosene at different levels. Positions of the SFS maxima and the fluorescence intensity of the leaded petrol and diesel shifted with the addition of kerosene. The magnitude of the shift and the change in fluorescence intensity are indicatives of the added kerosene. Some of the petrol and diesel samples purchased from different part of the country exhibited the char racteristics of the fuel adulterants. There is strong evidence that kerosene was used as an adulterant for some of these samples. Few of the samples exhibit different SFS fingerprints which are not characteristic for diesel, kerosene or leaded petrol. We are investigating different analytical parameters that can be utilised in quantifying the level of adulterant in these fuels. 